\title{
HEART RATE VARIABILITY (HRV) ANALYSIS IN RADIO AND TV BROADCASTING STATIONS WORKERS
}

\author{
ALICJA BORTKIEWICZ ${ }^{1}$, ELŻBIETA GADZICKA ${ }^{1}$, WIESŁAW SZYMCZAK ${ }^{3}$, \\ and MAREK ZMYŚLONY² \\ ${ }^{1}$ Nofer Institute of Occupational Medicine, Łódź, Poland \\ Department of Work Physiology and Ergonomics \\ ${ }^{2}$ Nofer Institute of Occupational Medicine, Łódź, Poland \\ Department of Radiological Protection \\ ${ }^{3}$ University of Łódź, Łódź, Poland \\ Department of Psychological Research Methodology and Statistics
}

\begin{abstract}
Objectives: The aim of the study was to assess the mechanism of cardiovascular impairments in workers exposed to UHF-VHF radio frequency electromagnetic fields (EMF). Materials and Methods: Heart rate variability (HRV) was analysed using 512 normal heart beats registered at rest. The analysis concerned time-domain (STD R-R) and frequencydomain (VLF, LF, HF) parameters of HRV. Fifty nine workers (group I) with low-level and 12 workers (group II) with highlevel exposure were examined. The mean age of the subjects was $47 \pm 9$ years and $41 \pm 14$ years, and mean exposure duration $19.1 \pm 8.8$ years and $13 \pm 4$ years, in groups I and II, respectively. The groups were divided according to: $\mathrm{E}_{\max }, \mathrm{E}_{\text {dose }}, \mathrm{E}_{\operatorname{mean}}$ for frequencies UHF, VHF and UHF+VHF: The control group consisted of 42 non-exposed subjects, aged $49 \pm 8$ years. Statistical analysis comprised one-way analysis of variance, covariance analysis and logistic regression models. Results: In the exposed groups, the heart rate was higher than in the control one. Standard deviation of R-R intervals (STD R-R) was found to be significantly $(\mathrm{p}=0.0285)$ lower in group I $(42.5 \pm 24.7 \mathrm{~ms})$ compared to the control group $(62.9 \pm 53.5 \mathrm{~ms})$. The risk of lowered STD R-R was significantly increased $(\mathrm{OR}=2.37, \mathrm{p}=0.023)$ in group II. Both exposed groups presented significantly higher VLF and LF values than the control group ( $\mathrm{p}=0.005$ and $\mathrm{p}=0.0025$, respectively). The EMF-exposed groups were characterised by the dominance of the sympathetic system (LF/HF $1.3 \pm 0.35)$. Conclusions: The results indicate that exposure to radiofrequency EMF may affect the neurovegetative regulation.
\end{abstract}

Key words:

Radio-frequency electromagnetic fields, Heart rate variability, Autonomic regulation, Broadcasting stations workers

This work was prepared partly as part of the project entitled: "Risk factors of work-related diseases - methods of evaluation and prevention" (REWARD) financed by European Union under the People Maria Curie Actions, International Research Staff Exchange Scheme, FP7 - People - IRSES - 2008 and partly as part of the project entitled: "Development of comprehensive projects for prevention of cardiovascular diseases" co-financed by European Union under the European Social Fund: Human Capital Programme (project no. WND-POKL.02.03.01-00-001/08).

Received: August 16, 2012. Accepted: November 5, 2012.

Address reprint request to A. Bortkiewicz, Department of Work Physiology and Ergonomics, Nofer Institute of Occupational Medicine, św. Teresy 8, 91-348 Łódź, Poland (e-mail: alab@bg.p.lodz.pl). 


\section{INTRODUCTION}

In recent years, there has been a rapid increase in the use of devices emitting radio and microwave $(100 \mathrm{kHz}-$ $300 \mathrm{GHz}$ ) electromagnetic fields EMF. These include the radio and television broadcasting stations, consumer devices such as microwave ovens and mobile phones. The rapid growth in the number of people exposed to radio- and microwave-frequency EMF has spurred an increasing interest in their impact on the human and possible adverse health effects. Although exposure levels attributable to those devices are below the maximum admissible levels specified by relevant regulations, it should be noted that these regulations are based on the anticipated thermal effects, while non-thermal effects have been ignored. Numerous studies are being currently undertaken aiming at clarification of the possible health effects of exposure to such EMF levels. It is believed that the autonomic nervous system which regulates the function of, among others, cardiovascular system is the system that may be sensitive to weak EMF.

Literature data on the impact of the radio-frequency EMF on the function of the autonomic nervous system and cardiovascular system is scarce and derives mainly from experimental studies [1]. Moreover, the results of these studies cannot be extrapolated to humans. The research team from the Nofer Institute of Occupational Medicine, Łódź, Poland, has continued for a couple of years projects regarding cardiovascular function and its neurovegetative regulation in workers occupationally exposed to different frequency EMF: at switchyard substations $(50 \mathrm{~Hz})[2,3]$, at medium frequency (MF) broadcasting stations $(1 \mathrm{MHz})$ [4], at high frequency (HF) communication centres $(10-50 \mathrm{MHz})$ and radioservices $(160 \mathrm{MHz})[5,6]$.

The resultant findings indicate that EMF exposure induces various cardiovascular abnormalities, of the types depending on EMF frequency. Exposure to MF EMF resulted in an increased number of abnormal ECG records, mostly ventricular heart rhythm disturbances, while exposure to power frequency EMF, in an increased percentage of the subjects with elevated blood pressure (BP) and disturbed day-night BP regulation. EMF can influence cardiovascular function by a number of pathways leading to cardiovascular diseases (CVD), promoting pathological processes and triggering acute cardiac events. However, the dysregulation of the autonomic nervous system seems to be the major adverse effect of the EMF exposure.

The aim of the study was to evaluate autonomic regulation of cardiovascular system in workers exposed to UHF (300-3000 MHz) - VHF (30-300 MHz) radio frequency EMF.

For the purpose of this study, the heart rate variability analysis has been performed as it allows for monitoring of the sympathetic and parasympathetic activity of the autonomic nervous system [7]. The method has become commonly applied in the diagnostics of diabetic neuropathy and the risk assessment of a sudden cardiac death in post-infarction patients as well as for screening other cardiovascular and neurological disorders [8-11]. It is also used in experimental physiological studies [12,13], but is rarely applied to individual examinations of people occupationally exposed to various occupational factors [14].

\section{MATERIALS AND METHODS}

The exposed group consisted of technical personnel and security service male workers, aged 28-66, with the period of work ranging from 3 to 35 years, who were qualified by the occupational health practitioners as capable of work at permissible EMF levels. The examinations were carried out in 71 workers at four Broadcast Stations, who were exposed to VHF and UHF EMF. The Broadcast Stations selected for the study operate at frequencies ranging from 66-727 MHz. These objects are characterised by permanent exposure of their workers to electromagnetic fields (mostly electric field). The main source of EMF are the transmitting antennas (half-wave dipole), 
radio transmitters and feeders (which conduct radio signals from the transmitter to the antenna). The duties of the Broadcast Station workers included operation of the broadcasting devices, control of the quality parameters and maintenance of broadcasting as scheduled. The exposed group was subdivided, according to exposure level, into two groups:

- Group I - low-level exposure - 59 subjects aged $47 \pm 9$ years, period of work: $19.1 \pm 8.8$ years.

- Group II- high-level exposure -12 subjects aged $41 \pm 14$ years, period of work: $13 \pm 4$ years.

Characteristiscs of exposure is displayed in Table 1.

The control group consisted of 42 workers of four Radio Link Stations (RLS) aged 33-64 years, mean 49 \pm , period of work $13 \pm 4$ years. Radio Link Stations are the elements of a telecommunication system in which signals are transmitted using EM waves focused into very narrow beams by directional (mostly parabolic) antennas. Due to the fact that the antennas are installed in highly inaccessible locations and that the radiation beams run high above the ground, the workers of the Radio Link Stations are free from exposure. The control group was similar to the exposed group in terms of the tasks to be performed and organization of work. As regards the exposed and control group, all the workers currently employed in a given organization included in the project were subjected to the examination. Characteristics of the examined groups is presented in Table 2.

All workers gave their formal consent prior to inclusion to the study. The protocol was approved by the Regional Biomedical Ethics Committee.

\section{METHODS}

The workers had the following performed: an interview including cardiological and family history, dietary habits, and leisure time activities, general medical examination with blood pressure measurement, heart rate variability (HRV) analysis.

To assess the neurovegetative regulation, resting heart rate variability was analysed. It is a sensitive method that enables assessment of the activity of the particular parts of the autonomic nervous system. An essential advantage of this method is that it is non-invasive and does not require active cooperation of the subject examined. HRV analysis was performed using the Medea system (Gliwice, Poland) from X, Y, Z orthogonal leads, when patient was in a horizontal position, after 10-min rest, during

Table 1. Characteristics of the study populations

\begin{tabular}{lccc}
\hline \multirow{2}{*}{ Parameters } & \multicolumn{2}{c}{ Exposed groups } & \multirow{2}{*}{ Group 0 (control) } \\
\cline { 2 - 3 } & I & II & 42 \\
Workers (n) & 59 & 12 & $49 \pm 8$ \\
Age (years) & $47 \pm 9^{*}$ & $44 \pm 12$ & $17 \pm 13$ \\
Period of employment (years) & $21 \pm 10$ & $18 \pm 11$ & \\
Medical interview (n, \%) & & & $8(19)$ \\
$\quad$ hypertension subjective & $8(14)$ & $3(25)$ & $15(29)$ \\
$\quad$ cardiovascular symptoms & $30(51)$ & $5(42)$ & $25 \pm 4$ \\
Body mass index & $27 \pm 4$ & $26 \pm 6$ & $17(40)$ \\
Smokers (> 10 cigarettes a day) (n, \%) & $24(41)$ & $6(50)$ & $25(59)$ \\
Subjects reporting alcohol consumption & $7(12)^{*}$ & $5(42)$ & \\
$\quad$ (not less frequently than once a month) $(\mathrm{n}, \%)$ & & & \\
\hline
\end{tabular}

* Statistically significant difference $(\mathrm{p}<0.05)$. 
Table 2. Exposure characteristics

\begin{tabular}{lccccccccc}
\hline \multirow{2}{*}{ Groups } & \multicolumn{3}{c}{$\begin{array}{c}\mathrm{E}_{\text {dose }} \\
\left(\mathrm{V}^{2} \mathrm{~h} / \mathrm{m}^{2}\right)\end{array}$} & \multicolumn{3}{c}{$\begin{array}{c}\mathrm{E}_{\text {mean }} \\
(\mathrm{V} / \mathrm{m})\end{array}$} & & \multicolumn{3}{c}{$\begin{array}{c}\mathrm{E}_{\max } \\
(\mathrm{V} / \mathrm{m})\end{array}$} \\
\cline { 2 - 10 } & UHF & VHF & UHF_VHF & UHF & VHF & UHF_VHF & UHF & VHF & UHF_VHF \\
\hline Group I & & & & & & & & & \\
$\quad$ M & 288 & 6570 & 6848 & 0.1 & 0.3 & 0.3 & 5.3 & 0.9 & 7.9 \\
$\quad$ SD & 304 & 8848 & 9026 & 0.1 & 0.3 & 0.3 & 6.9 & 0.7 & 5.7 \\
Group II & & & & & & & & & \\
$\quad$ M & 130130 & 640583 & 771200 & 1.1 & 2.5 & 2.7 & 6.8 & 15.2 & 16.7 \\
$\quad$ SD & 86116 & 423505 & 509990 & 0.1 & 0.1 & 0.1 & 0.0 & 0.0 & 0.0 \\
Statistical significance (p) & 0.0001 & 0.0001 & 0.0001 & 0.0001 & 0.0001 & 0.0001 & 0.0001 & 0.0001 & $>0.05$ \\
\hline
\end{tabular}

$\mathrm{E}_{\mathrm{dose}}-$ lifetime dose of $\mathrm{E}$, calculated from the history of employment and job timetable.

$\mathrm{E}_{\text {mean }}-$ mean value of $\mathrm{E}$.

$\mathrm{E}_{\max }-$ maximum value of the electric field strength.

UHF - ultra high frequency $(300-3000 \mathrm{MHz})$.

VHF - very high frequency $(30-300 \mathrm{MHz})$.

$\mathrm{M}$ - mean value, $\mathrm{SD}$ - standard deviation.

spontaneous breathing. Considering that HRV parameters were significantly affected by the time of the day, intake of food, drugs or condiments, etc., the examinations were performed in standard conditions. The workers were examined before work, $2 \mathrm{~h}$ after a light meal. Before the examination, the patients refrained from the intake of drinks containing caffeine, theine, or alcohol and from smoking tobacco. None of the examined subjects took drugs which might affect autonomic nervous system (such as e.g. beta blockers). Five hundred twelve consecutive, normal cardiac evolutions (cycles) were registered under resting conditions.

ECG signal is sampled at a rate of 2000 samples per sec. and filtered to remove ectopic activity and artefacts prior to R-R interval file generation. The system has a high accuracy of QRS detection. The collected short-term ECG records were used to determine time- and frequency-domain parameters. Statistical analysis made it possible to calculate the following: mean R-R interval; R-R standard deviation; median, modal, minimum and maximum R-R values. ECG records were also subjected to the Fast Fourier Transformation (FFT) using the Blackman-Harris window. The power spectrum density (area covered by the power spectrum) was computed for the following frequency bands: very low (VLF): 0.0167-0.05 Hz; low (LF): $0.05-0.15 \mathrm{~Hz}$; high (HF): 0.15-0.35 Hz; ultra high (UHF): $0.35-0.50 \mathrm{~Hz}$, and expressed as a percentage of the total power spectrum calculated as the sum of the power spectra for $0.0167-0.5 \mathrm{~Hz}$ frequency range. According to the commonly adopted interpretation, the high-frequency power spectrum (HF) is parasympathetic-mediated, while the low-frequency one (LF) is sympathetic/parasympathetic- mediated. The LF/HF ratio indicates sympathovagal balance [7].

\section{Exposure evaluation}

Assessment of exposure has been based on a spectral analysis for a typical broadcasting station in the UHF and VHF bands and on the specifications of the apparatus installed there. To assess individual exposure to EMF, the following parameters were calculated separately for VHF, UHF and $\mathrm{VHF}+\mathrm{UHF}$ :

- maximum value of the electric field strength $\left(\mathrm{E}_{\mathrm{max}}\right)$,

- mean value of $\mathrm{E}\left(\mathrm{E}_{\text {mean }}\right)$, 
- lifetime dose of $\mathrm{E}\left(\mathrm{E}_{\text {dose }}\right)$, calculated from the history of employment and job timetable.

The method for the assessment of these values has been presented elsewhere [15].

\section{Statistical analysis}

The results were analysed using the following methods: chi-square test (for proportions) and Student t-test (for normal distributions), or non-parametric Mann Whitney test for other distributions, to analyse differences between groups, Fisher exact test to compare the prevalence of cardiovascular abnormalities, covariance analysis to assess the effect of exposure on the continuous variables, while eliminating the confounding factors (age, tobacco smoking, alcohol consumption etc.), logistic regression models to calculate the relative risk of cardiovascular impairments as the effect of exposure and/or confounding factors.

For all of the used statistical tests significance level $\alpha=0.05$ was established. For analysis the software: SPSS version12 was used.

\section{RESULTS}

Out of 71 of the exposed subjects, $36(51 \%)$ workers reported cardiovscular symptoms, including dyspnoea, pain or discomfort in the chest or feeling of irregular heart beat. In the control group, similar complaints were reported by 15 (29\%) workers. During medical examination, 24 (34\%) people from the exposed group and 8 (19\%) from the control group had elevated arterial blood pressure.

The data from the medical examinations and the interview indicates that the examined groups were similar in respect to the level of physical activity and the dietary habits. They differed in terms of age, smoking habit and alcohol consumption. The possible influence of confounders on the study results was eliminated using statistical methods.

In the exposed and control groups, the mean standard deviation of R-R intervals (STD R-R) was high, which would indicate a normal heart rate variability. However, statistically significant differences between the groups were found in terms of STD R-R, which was lower

Table 3. Time-domain parameters of heart rate variability

\begin{tabular}{lcccccc}
\hline \multirow{2}{*}{ Groups } & \multicolumn{5}{c}{ Heart rate variability (ms) } \\
\cline { 2 - 6 } & STD R-R & AVG R-R & MED & MOD & Min & Max \\
\hline Group I & & & & & & \\
M & 42.46 & 861.07 & 864.15 & 855.17 & 735.56 & 989.97 \\
SD & 24.70 & 123.76 & 123.93 & 120.49 & 91.03 & 163.16 \\
Group II & & & & & & \\
M & 44.08 & 851.75 & 851.42 & 849.21 & 700.83 & 999.08 \\
SD & 25.81 & 95.40 & 96.51 & 121.68 & 115.33 & 132.18 \\
Group 0 & & & & & & \\
M & 62.90 & 864.12 & 867.46 & 859.78 & 687.73 & 1031.34 \\
SD & 53.50 & 126.37 & 128.28 & 132.47 & 123.55 & 149.33 \\
Statistical significance (p) & 0.0285 & $>0.05$ & $>0.05$ & $>0.05$ & $>0.05$ & $>0.05$ \\
& $(0$ vs. 1$)$ & & & & & \\
\hline
\end{tabular}

AVG R-R - average value of R-R (beat to beat).

STD R-R - standard deviation of R-R.

MED - median value, MOD - modal value.

Other abbreviations as in Table 2. 
Table 4. Frequency-domain parameters of heart rate variability

\begin{tabular}{lrrrrc}
\hline \multirow{2}{*}{ Groups } & \multicolumn{5}{c}{ Power in bands (\% of total power) } \\
\cline { 2 - 5 } Group I & VLF & LF & HF & TPS & LF/HF \\
M & & & & \\
SD & 14.60 & 29.28 & 23.30 & 74.11 & 1.31 \\
Group II & 3.67 & 5.94 & 5.27 & 6.21 & 0.35 \\
M & & & & \\
SD & 13.80 & 27.50 & 24.60 & 72.37 & 0.00 \\
Group 0 & 5.40 & 7.60 & 6.50 & 5.68 & 0.50 \\
M & & & & 71.20 & 0.20 \\
SD & 12.85 & 24.35 & 26.07 & 9.01 & 0.0016 \\
Statistical significance (p) & 3.88 & 5.03 & 3.77 & $>0.05$ & $(0$ vs. I, I vs. II) \\
\hline
\end{tabular}

VLF - very low frequency $(0.0167-0.05 \mathrm{~Hz})$.

LF - low frequency $(0.05-0.15 \mathrm{~Hz})$.

$\mathrm{HF}$ - high frequency $(0.15-0.35 \mathrm{~Hz})$.

TPS - total power spectrum.

Table 5. Relative risk of cardiovascular abnormalities in relation to EMF exposure in the UHF band, VHF band, UHF+VHF band

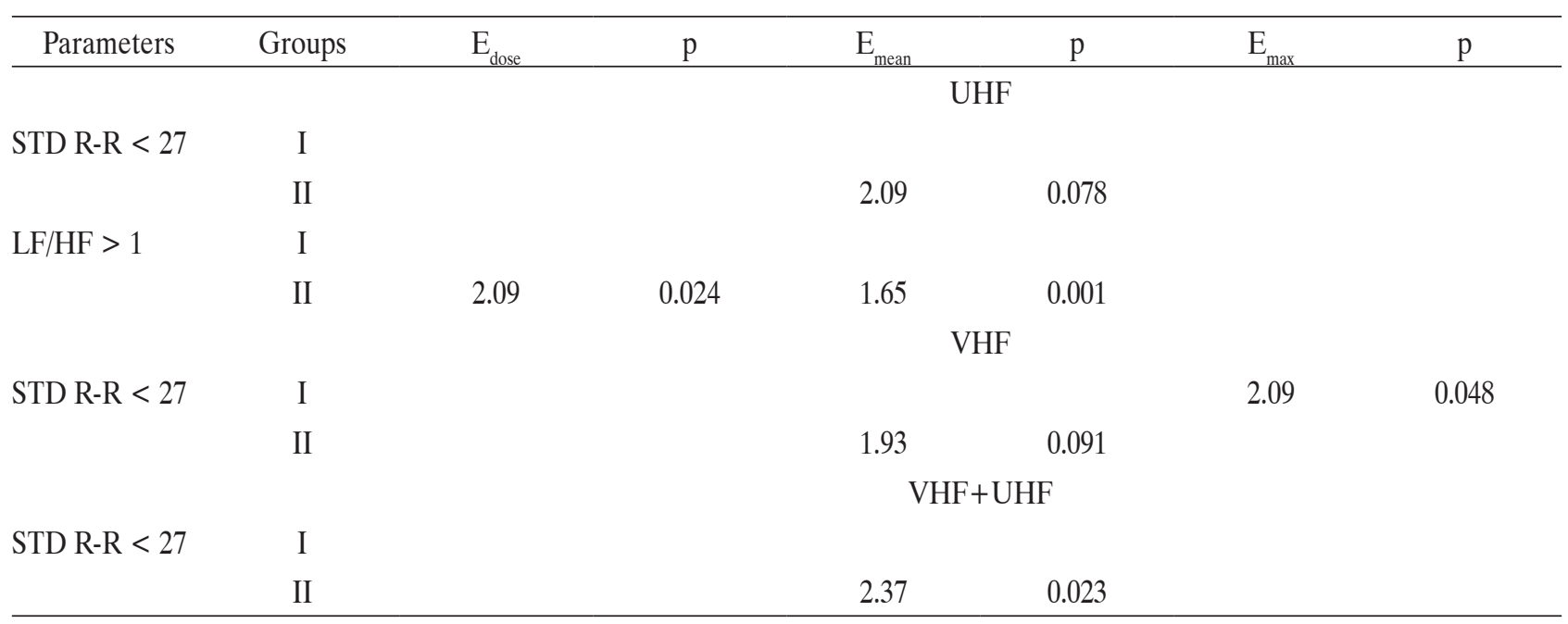

Abbreviations as in Table 2 and 4.

in the exposed groups. The results of the analysis of timedomain parameters are displayed in Table 3.

In the EMF-exposed groups, the percentage of subjects with decreased HRV (STD R-R $<27 \mathrm{~ms}$ ) was twice as high as in the control group $(\mathrm{p}=0.0285)$. In 18 subjects from group I (30.5\%) and three subjects from group II (25\%), the STD R-R was found to be lower than $27 \mathrm{~ms}$, which was adopted as the limit value [6]. A spectral analysis using FFT method revealed that VLF and LF were significantly higher in the exposed than in the control group. 
No significant differences in HF and TPS (total power spectrum) were detected between the exposed and control groups. LF/HF ratio was significantly higher in the exposed groups. In both exposed groups, the sympathetic activity dominated.

The details of power spectrum analysis for all the study groups are presented in Table 4.

In order to investigate whether the impaired neurovegetative regulation was associated with EMF exposure, the risk of such impairments was assessed in relation to the level of EMF exposure in the UHF, VHF and UHF+VHF bands. The reference was the control group for whom the risk of neurovegetative impairments was assumed to be the unity. The analysis revealed that the impaired neurovegetative regulation correlated with selected parameters of EMF exposure. Detailed data is displayed in Table 5.

\section{DISCUSSION}

The HRV analysis revealed a higher resting heart rate (lower AVG R-R) in the subjects exposed to UHFVHF EMF. The increased resting heart rate is an unfavourable effect. As revealed by the Framingham study, in such subjects, the risk of a sudden cardiac death is $30 \%$ higher than in the subjects with a lower heart rate [16]. In the subjects exposed to UHF-VHF EMF, the STD R-R was found to be lower. The percentage of the subjects with critically decreased HRV (STD R-R $<27 \mathrm{~ms}$ ) was twice as high in the exposed group as in the control group. The lowered HRV may also lead to some negative consequences. A number of prospective studies indicate that for patients with cardiovascular abnormalities in $24 \mathrm{~h}$ ECG monitoring, which are accompanied by a decreased HRV, the risk of a sudden cardiac death is twice as high as in patients with similar ventricular arrhythmias but normal HRV value [17]. The risk of a sudden cardiac death is higher also in the healthy subjects with lower HRV [18].
Our analysis of the frequency-domain parameters revealed that in the exposed groups the power spectrum in the VLF band had been significantly higher than in the control group. However, the interpretation of the significance of changes within the power spectrum in this band is very difficult. Scarce information is available on the slowest (VLF below $0.05 \mathrm{~Hz}$ ) component; it is supposed to be affected by both parts of the autonomic nervous system as well as the thermoregulatory processes and the renin/angiotensin system [12,13]. The latter relationship is, according to some authors, disputable. The renin-angiotensis system plays a significant role in the pathogenesis of arterial hypertension, with angiotensin II acting on the blood vessels [19]. An increase in the power spectrum in the VLF band would indicate that one of the mechanisms for the increased arterial blood pressure may be connected with the influence of EMF on the renin-angiotensin system.

Another one seems to be an activation of the sympathetic system, the activity of which is described by the LF component. LF was found to be higher in the exposed groups than in the control group. In the exposed groups blood pressure changes were also more prevalent (34\% vs. $19 \%$ ). Also in the exposed groups, the indicator of neurovegetative balance (LF/HF ratio) was significantly higher compared to the control group. This would point to the dominance of the sympathetic activity in the neurovegetative regulation of the cardiovascular function. The predominant sympathetic activity and the resulting decrease in the cardioprotective activity of the parasympathetic system makes the organism more prone not only to hypertension but also to the heart rhythm disturbances and the development of the coronary heart disease [20,21]. Neurovegetative disturbances were also found in our earlier study in workers exposed to $160 \mathrm{MHz}$ electromagnetic fields. In this group, the HRV changes were reflected by the power spectrum in the LF and VLF bands and correlated with those in arterial blood pressure [22]. As regards the workers exposed to medium frequency EMF, statistically significant negative correlation was found between the maximum intensity of EMF and HF power spectrum [4]. 
It is difficult to compare our findings on neurovegetative regulation in workers exposed to VHF-UHF electromagnetic fields with relevant literature reports since most of the studies employed different methodology, which allows for a less detailed analysis. However, the nature of most of the reported subjective symptoms implies their association with impaired neurovegetative regulation of the cardiovascular function [23,24]. In the experimental study by Mann and Roschke, the HRV analysis did not reveal any pathologies in the subjects exposed to pulse-modulated 900 MHz EMF [25]. Barutcu et al. (2011), Parazzini et al. (2007), Barker et al. (2007) likewise found no HRV changes during experimental exposures to EMF from mobile phones [26-28]. Results of the studies performed by Andrzejak et al. (2008) indicate that during the call by mobile phone parasympathetic activity (HF), measured by the use of analysis of heart rate variability, increased and sympathetic activity (LF) decreased [29].

However, as in all experimental studies, only the immediate responses to exposure were observed. It did not allow for monitoring of the delayed effects related to long-term exposure $[25,30]$ which is most prevalent in the occupational setting.

Our results are connected with long-term exposure. Results demonstrating the influence of the UHF EMF on the increased activity of the sympathetic part of the autonomic nervous system seem to be particularly interesting (Table 5). The significance of this observation stems from the fact that the UHF frequencies $(30 \mathrm{MHz}-3 \mathrm{GHz})$ include the frequency range of mobile telephone systems. Therefore, it is reasonable to expect that the mechanism of biological activity of EMF emitted by transmitting antennas of base stations and mobile TV transmitters may be similar. This could explain subjective symptoms from the central nervous system (such as headache and dizziness) reported by the residents of areas surrounding base stations, observed by different authors [23,24,31-37] where EMF with similar values prevail.
However, please note that, although the levels of electric field are both quite similar, the EMF spectrum in the exposed people is different (in case of the analyzed transmission facilities, in addition to the UHF EMF there is an additional VHF EMF component). Also, EMF operating frequencies are not identical (although they all are the parts of the same UHF range). It seems that further intensive research on the impact of UHF-frequency EMF on the neurovegetative regulation of cardiovascular system in people living around base stations is necessary. This could explain the origin of the reported symptoms.

\section{CONCLUSIONS}

Although in the present study the level of EMF exposure was rather low and did not exceed the limits specified by relevant Polish hygienic standards, a number of impairments in neurovegetative regulation could be found in the groups of the exposed workers. These were expressed as significantly decreased HRV (STD R-R < $27 \mathrm{~ms}$ ) ( $\mathrm{p}=0.0285)$, increased power spectrum within the $\operatorname{VLF}(\mathrm{p}=0.005)$ and $\mathrm{LF}$ $(p=0.0025)$ bands, predominance of the sympathetic activity $(\mathrm{LF})$ in the neurovegetative regulation $(\mathrm{LF} / \mathrm{HF}=1.3 \pm 0.35)$. These impairments were found to significantly correlate with exposure parameters, mostly $\mathrm{E}_{\text {mean }}$ and, to a lesser extent, with the lifetime dose. Therefore, it seems reasonable to consider, whether the exposure assessment of workers exposed to EMF in the UHF+ VHF band(s) should be limited to $\mathrm{E}_{\text {max }}$ only, or should also include such parameters as $\mathrm{E}_{\text {mean }}$ and the lifetime dose. Current regulations on the workplace hygiene provide for assessment of EMF dose only in case of work in the hazard zone.

Our results suggest that similar changes in the neurovegetative regulation are likely to occur in the inhabitants of the areas located close to the mobile phone base stations, as the intensities of the UHF EMF to which they are exposed are very similar to those affecting the workers of the broadcasting stations. 


\section{REFERENCES}

1. Repacholi MH. Low level exposure to radiofrequency electromagnetic fields. Health effects and research needs. Bioelectromagnetics 1998;19(1):1-19.

2. Bortkiewicz A, Zmyslony M, Palczynski C, Gadzicka E, Szmigielski St. Dysregulation of autonomic control of cardiac function in workers at AM broadcast stations (0.738-1.503 MHz). Electro- and Magnetobiol 1995;14(3):177-91.

3. Bortkiewicz A, Gadzicka E, Zmyślony M, Szymczak W. Neurovegetative disturbances in workers exposed to $50 \mathrm{~Hz}$ electromagnetic fields. Int J Occup Med Environ Health 2006;19(1): 53-60.

4. Bortkiewicz A, Gadzicka E, Zmyslony M. Heart rate variability in workers exposed to medium-frequency electromagnetic fields. J Auton Nerv Syst 1996;59(3):90-7.

5. Bortkiewicz A, Zmyslony M, Gadzicka E, Palczynski C. Ambulatory ECG monitoring in workers exposed to MF electromagnetic fields. J Med EngTechnol 1997;21(2):41-6.

6. Bortkiewicz A, Gadzicka E, Zmyślony M, Szymczak W. Impaired neurovegetative regulation in radio and tv broadcasting station workers: HRV analysis. In: Kostarakis $\mathrm{P}$, editor. Biological effects of EMFs. Proceedings of the 3rd International Workshop; 2004 Oct 4-8, Kos, Greece, Vol. 2. University of Ioannina; 2004, p. 1049-54.

7. Malik M. Heart rate variability. Standards of measurement. Physiological interpretation and clinical use. Circulation 1996;93(5):1043-65.

8. Binkley PF, Nunziata E, Haas GJ, Nelson SD, Cody RJ. Parasympathetic withdrawals an integral component of autonomic imbalance in congestive heart failure: demonstration in human subjects and verification in a paced canine model of ventricular failure. J Am Coll Cardiol 1991;18(2):464-72.

9. Muhlnickel B. The value of heart rate frequency variability in the prognostic evaluation of patients with severe cerebral injuries. Anaesthesiol Reanim 1990;15(6):342-50.

10. Hayano J, Yamada A, Mukari S, Sakakibara Y, Yamada M, Ohte N, et al. Severity of coronary atherosclerosis correlates with the respiratory component of heart rate variability. Am Heart J 1991;121(4 Pt 1):1070-9.

11. Van Ravenswaaij-Arts CM, Kollee LA, Hopman JC, Stoelinga GB, Van Geijn HP. Heart rate variability. Ann Int Med 1993;118(6):436-47.

12. Trzebski A, Smietanowski M. Cardiovascular periodicities in healthy humans in the absence of breathing and under reduced chemical drive of respiration. J Auton Nerv Syst 1996;57(3):144-8.

13. Brenner IK, Thomas S, Shephard RJ. Autonomic regulation of the circulation during exercise and heat exposure. Inferences from heart rate variability. Sports Med 1998;26(2):85-99.

14. Kristal-Boneh E, Reifel M, Froom P, Ribak J. Heart rate variability in health and disease. Scand J Work Environ Health 1995;21(2):85-95.

15. Zmyślony M, Aniołczyk H, Bortkiewicz A. Exposure to VHF and UHF electromagnetic fields among workers employed in radio and TV broadcast centers. I. Exposure assessment. Med Pr 2001;52(5):321-7 [in Polish].

16. Kannel WB, Kannel C, Paffenberger RS, Jr, Cupples LA. Heart rate and cardiovascular mortality. The Framingham Study. Am Heart J 1987;113(6):1489-94.

17. Singer DH, Martin GJ, Magid N, Weiss JS, Schaad JW, Kechoe $\mathrm{R}$, et al. Low heart rate variability and sudden cardiac death. J Electrocardiol 1988;21(Suppl):S46-55.

18. Huikuri HV, Castellanos A, Myerburg RJ. Sudden death due to cardiac arrhythmias. N Engl J Med 2001;345(20):1473-82.

19. Griffin SA, Brown WC, MacPherson F, MacGrath JC, Wilson VG, Korsgaard N, et al. Agiotension II causes vascular hypertrophy in part by non-pressor mechanisms. Hypertension 1991;17(5):626-35.

20. Hayano J, Sakakibara Y, Yamada M, Ohte N, Fujinami T, Yokoyama K, et al. Decreased magnitude of heart rate spectral components in coronary artery disease. Circulation 1990;81(4):1217-24.

21. Guzzetti S, Piccaluga E, Casati R, Cerutti S, Lombard F, Pagani M, et al. Sympathetic predominance in essential 
hypertension: a study employing analysis heart rate variability. J Hypertens 1988;6(9):711-7.

22. Bortkiewicz A, Gadzicka E, Zmyslony M, Szymczak W. Neurovegetative disturbances in workers exposed to elektromagnetic fields. In: Magjarevic R, Tonkovic S, Bilas V, Lackovic I, editors. Proceedings of the International Federation for Medical \& Biological Engineering IX Mediterranean Conference on Medical and Biological Engineering and Computing Medicon 2001. June 12-15, Pula, Croatia 2001, p. 769-72.

23. Bortkiewicz A, Gadzicka E, Szyjkowska A, Politański P, Mamrot P, Szymczak W, et al. Subjective complaints of people living near mobile phone base stations in Poland. Int J Occup Med Environ Health 2012;25(1):31-40. DOI 10.2478/ s13382-012-0007-9.

24. Khurana VG, Hardell L, Everaert J, Bortkiewicz A, Carlberg M, Ahonen M. Epidemiological evidence for a health risk from mobile phone base stations. Int J Occup Environ Health 2010;16(3):263-7.

25. Mann K, Roschke J. Effects of pulsed high frequency electromagnetic fields on human sleep. Neuropsychobiology 1996;33(1):41-7.

26. Barutcu I, Esen AM, Kaya D, Turkmen M, Karakaya O, Saglam M, et al. Do mobile phones pose a potential risk to autonomic modulation of the heart? Pacing Clin Electrophysiol 2011;34(11):1511-4.

27. Parazzini M, Ravazzani P, Tognola G, Thuróczy G, Molnar FB, Sacchettini A, et al. Electromagnetic fields produced by GSM cellular phones and heart rate variability. Bioelectromagnetics 2007;28(2):122-9.

28. Barker AT, Jackson PR, Parry H, Coulton LA, Cook GG, Wood SM. The effect of GSM and TETRA mobile handset signals on blood pressure, catechol levels and heart rate variability. Bioelectromagnetics 2007;28(6):433-8.

29. Andrzejak R, Poreba R, Poreba M, Derkacz A, Skalik R, $\mathrm{Gac} \mathrm{P}$, et al. The influence of the call with a mobile phone on heart rate variability parameters in healthy volunteers. Ind Health 2008;46(4):409-17.

30. Braune S, Wracklage C, Raczek J, Lucking CH. Resting blood pressure increase during exposure to a radio-frequency electromagnetic field. Lancet 1998;351(9119):1857-8.

31. Santini R, Santini P, Danze JM, Le Ruz P, Seigne M. Study of the health of people living in the vicinity of mobile phone base stations: I. Influences of distance and sex. Pathol Biol 2002;50:369-73.

32. Santini R, Santini P, Le Ruz P, Danze JM, Seigne M. Survey study of people living in the vicinity of cellular phone base stations. Electromag Biol Med 2003;1:41-9.

33. Navarro EA, Segura J, Portoles M, Gomez-Peretta C. The Microwave syndrome: A preliminary study in Spain. Electromag Biol Med 2003;22:161-9.

34. Hutter HP, Moshamer H, Wallner P, Kundi M. Subjective symptoms, sleeping problems and cognitive performance in sub-jects living near mobile phone base stations. Occup Environ Med 2006;63:307-13.

35. Abdel-Rassoul G, El-Fateh OA, Salem MA, Michaele A, Farahat F, El-Batanomy M, et al. Neurobehavioral effects among inhabitants around mobile phone base stations. Neurotoxicology 2007;28:434-40.

36. Berg-Beckhoff G, Blettner M, Kowall B, Breckenkamp J, Schlehofer B, Schmiedel S, et al. Mobile phone base stations and adverse health effects: Phase 2 of a cross-sectional study with measured radio frequency electromagnetic fields. Occup Environ Med 2009;66:124-30.

37. Moser M, Röösli M. Epidemiological studies on mobile phone base stations and health. Possible biological out-comes and study designs. Cost 281: Workshop on Mobile Phone base Stations and Health, 2003 May 15-16; Dublin, Ireland [cited 2003 Aug 15]. Available from URL: http://www.radio.fer/ cost281.

This work is available in Open Access model and licensed under a Creative Commons Attribution-NonCommercial 3.0 Poland License - http://creativecommons.org/ licenses/by-nc/3.0/pl/deed.en. 